\begin{tabular}{ll|l}
\cline { 2 - 3 } & \multicolumn{2}{l}{ Intervent Neurol 2016;5:123-130 } \\
\cline { 2 - 3 } & $\begin{array}{l}\text { DOI: 10.1159/000447022 2016 S. Karger AG, Basel } \\
\text { Published online: June 16, } 2016\end{array}$ & $\begin{array}{l}\text { 1664-9737/16/0054-0123\$39.50/0 } \\
\text { www.karger.com/ine }\end{array}$ \\
\hline
\end{tabular}

\title{
Safety Outcomes Using a Proximal Protection Device in Carotid Stenting of Long Carotid Stenoses
}

\author{
Kunakorn Atchaneeyasakul Priyank Khandelwal Sudheer Ambekar \\ Kevin Ramdas Luis Guada Dileep Yavagal \\ Interventional Division, Department of Neurology, University of Miami Miller School of \\ Medicine, Miami, Fla., USA
}

\section{Key Words}

Carotid stenosis · Carotid stent · Microemboli $\cdot$ Proximal protection $\cdot$ Stroke

\begin{abstract}
Background: Embolic protection devices can prevent atherosclerotic emboli during carotid stenting. Newer proximal protection devices reverse flow in the internal carotid artery (ICA), leading to reduction in perioperative microemboli. The risk of stroke is high for carotid stenting of ICA lesions with a length $>10 \mathrm{~mm}$ and/or angiographic string sign. Objective: We aimed to evaluate the safety outcomes of proximal embolic protection device usage in this high-risk group. Methods: This is a retrospective analysis of patients who underwent carotid stenting procedures with proximal embolic protection devices at a tertiary care center. High-risk features for adverse events with carotid stenting were identified. Peri- and postprocedural outcomes were recorded. We further compared outcomes in patients with a carotid stenosis length $>10$ mm to those with shorter stenosis. Results: From January 2011 to December 2014, we included 27 patients; $96.3 \%$ were symptomatic and 3.7\% were asymptomatic. There was a stent placement technical success rate of $100 \%$. No major stroke or coronary events were recorded. One minor stroke event developed in one patient. A carotid lesion length $>10 \mathrm{~mm}$ and/or angiographic string sign was noted in 21/27 patients, with an average lesion length of $14.4 \mathrm{~mm}$. One patient (4.8\%) in this group developed a minor stroke event. Neither a coronary nor a major stroke event was recorded in this group. There was no significant difference in the complication rate between the long lesion and the control group. Conclusion: In our patient cohort, it was found that a proximal embolic protection device is safe for patients with carotid stenosis, including those with a carotid lesion length $>10 \mathrm{~mm}$ and/or angiographic string sign.




\section{Introduction}

The annual incidence of acute ischemic stroke in the United States is approximately 795,000; of these cases, 185,000 are recurrent strokes [1]. One of the major causes of stroke is large artery atherosclerotic disease of the internal carotid artery (ICA). The secondary prevention of strokes that are due to this cause can be established by revascularization of the carotid artery, which is achieved by carotid endarterectomy or carotid artery stenting (CAS). Perioperative microembolism is a potential adverse event of these revascularization techniques, and it is more common with CAS than with carotid endarterectomy [2]. An embolic protection device is commonly utilized with CAS with the aim of preventing atherosclerotic debris from becoming dislodged during the intervention. However, distal embolic protection devices, also known as filtering devices, may not be able to prevent all perioperative emboli from flowing distally, either due to the filter traversing through an unprotected atherosclerotic lesion, or given the presence of microemboli that are smaller than the filter pores [3]. Thus, the effectiveness of these devices stands to be improved.

Newer proximal protection devices utilize the ICA flow reversal method by occluding the external carotid artery (ECA) and the common carotid artery (CCA). Several studies have shown proximal protection devices to significantly reduce the perioperative microembolic signal detected by transcranial Doppler, and they also decrease the number of new ischemic lesions when compared to distal protection devices [4-6]. Traditionally, patients with a carotid lesion length $>10 \mathrm{~mm}$ and/or angiographic string sign, a history of prior stroke or coronary artery disease, contralateral carotid stenosis $>50 \%$, older age, and female sex were reported to have a higher percentage of adverse outcomes and were also considered high-risk groups [7-9]. The outcome of carotid stenting in these particular groups with the use of proximal protection devices is not well studied. We aimed to evaluate the outcome of stenting with proximal embolic protection in these groups.

\section{Methods}

This is a retrospective analysis of consecutive patients who underwent a carotid stenting procedure with the placement of a proximal embolic protection device at our tertiary care academic medical center from January 2011 to December 2014. This study was approved by our institutional review board. Patient data were obtained from our electronic patient database. All patients who received ICA stenting with a proximal embolic protection device were included in this investigation. Information allowing patient identification was removed from this study.

Patients' demographic information, including age at the time of the carotid stenting procedure, sex, and atherosclerotic risk factors (including hypertension, hyperlipidemia, diabetes, recent HbA1c level, smoking history, body mass index, and prior coronary artery disease or stroke) was documented. The presenting symptoms that led to the diagnosis of carotid stenosis are listed. Procedural data, including procedure duration and the time from procedure start to successful stent placement, were collected. Postprocedural data including residual carotid stenosis and outcomes at discharge were also included. Modified Rankin Scale (mRS) scores, which were obtained prior to the procedure and on the date of discharge from the hospital, were assessed from physical therapist and physician evaluation notes.

Carotid stenosis lesion characteristics were identified via carotid digital subtraction angiography images that were obtained from our institution's picture archiving and communication system (PACS). Stenosis location, lesion length, stenosis percentage, and the status of the contralateral carotid artery were evaluated with the Philips iSite Enterprise PACS toolsets (Philips Healthcare Informatics, Foster City, Calif., USA). The North American Symptomatic Carotid Endarterectomy Trial (NASCET) carotid stenosis measurement method, which compares the lumen size at the area with the greatest stenosis with the lumen size of the normal distal cervical ICA, was used to measure the degree of stenosis [10,11]. Angiographic string sign lesion stenosis was measured via the same methods. We were able to evaluate the normal distal cervical ICA for calculation in every patient. 


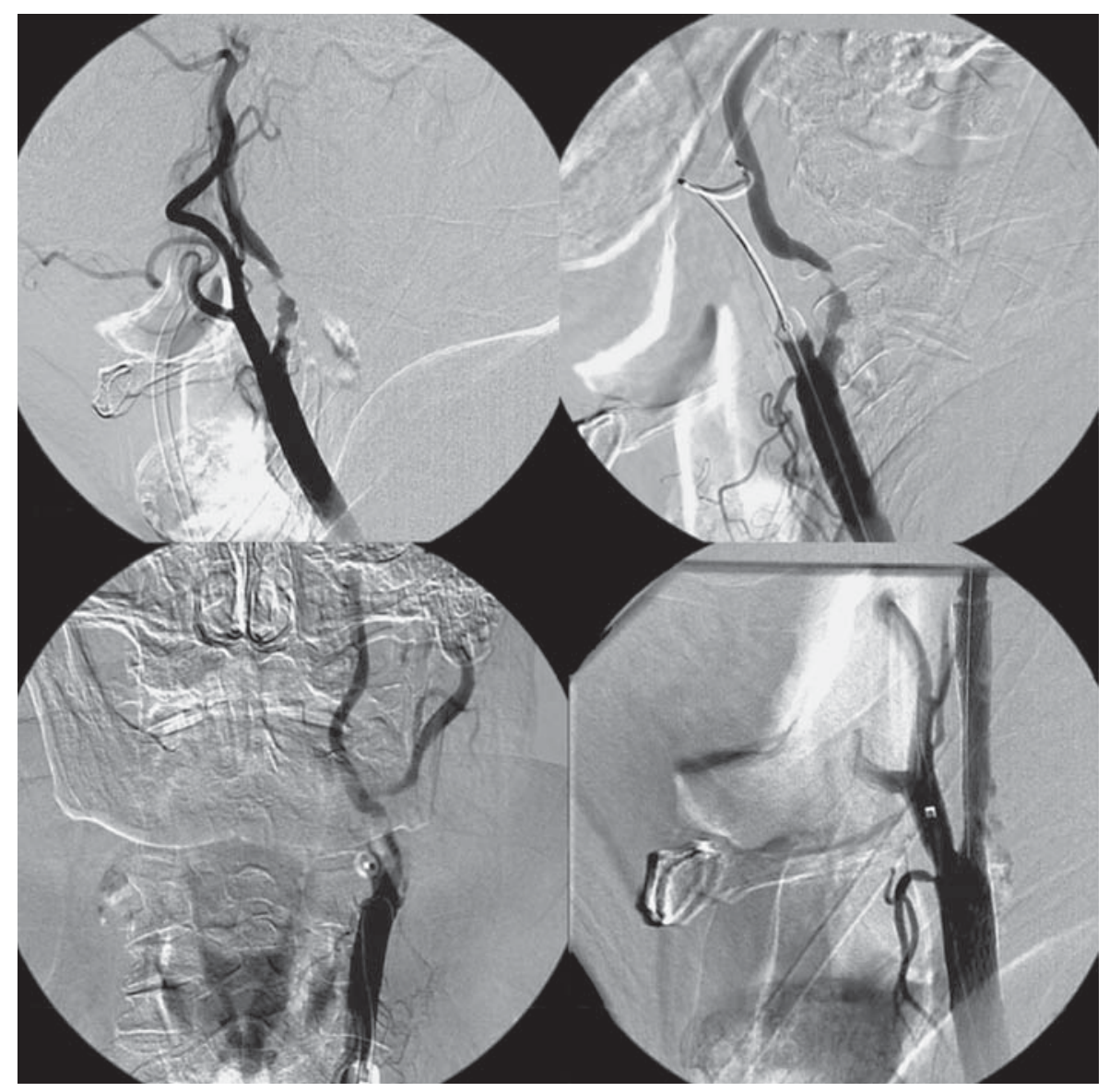

Fig. 1. Digital subtraction angiography images during the use of a proximal protection device in CAS. Upper left: visualization of long internal carotid stenosis. Upper right: distal balloon inflation in the ECA. Lower left: proximal balloon inflation in the CCA. Lower right: successful deployment of the internal carotid stent.

\section{Procedure Details}

CAS was performed using the Mo.Ma Ultra proximal cerebral protection device (Medtronic, Minneapolis, Minn., USA). All patients were placed on dual antiplatelet therapy at least 1 week prior to the procedure. All procedures were performed under conscious sedation. A 9-French sheath was inserted into the femoral artery and a diagnostic angiogram of the carotid arteries was performed to confirm the degree and location of the occlusion. The diagnostic catheter was then navigated into the ECA. The catheter was subsequently exchanged for the Mo.Ma guide catheter using a 0.035-inch Amplatz wire (Cook Medical, Bloomington, Ind., USA). The guide catheter of the Mo.Ma device was positioned in the ECA, such that the distal balloon covered the origin of the superior thyroid artery or was positioned proximal to it. The proximal balloon lay in the CCA. The distal balloon was inflated using 1:1 contrast (Visipaque 320; GE Healthcare, Cork, Ireland) and saline solutions. An angiogram was performed through the central lumen of the catheter to confirm occlusion of the ECA. Then, the proximal balloon was inflated to achieve antegrade flow arrest. Under roadmap guidance, with both balloons inflated, the site of stenosis in the ICA was crossed using a 0.014-inch microwire. Pre-balloon angioplasty was performed using a 3-mm monorail Maverick balloon if the stenosis was tight and crossing the lesion with the wire was not possible otherwise. The stent was deployed across the stenotic area. Post-balloon angioplasty was performed using a 5-mm Sterling balloon. It is essential that blood-containing debris is aspirated through the central lumen of the Mo.Ma catheter after post-balloon angioplasty until there is no more debris in two consecutive aspirates. It is also critical that the balloons remain inflated in the ECA and the CCA while traversing the lesion, as well as during pre-balloon angioplasty, stent deployment, and post-balloon angioplasty, to prevent distal flow of debris resulting in embolism of the distal branches. The ECA balloon was then deflated, followed by the CCA balloon. Figure 1 illustrates the utilization of a proximal protection device in CAS. 
Table 1. Baseline characteristics of the 27 patients undergoing CAS with a proximal protection device

\begin{tabular}{|c|c|}
\hline \multicolumn{2}{|l|}{ Sex } \\
\hline Male & $74.1 \%$ \\
\hline Female & $25.9 \%$ \\
\hline Age, years & $65.4 \pm 2.7$ \\
\hline \multicolumn{2}{|l|}{ Medical history } \\
\hline Hypertension & $92.3 \%$ \\
\hline Diabetes mellitus & $34.6 \%$ \\
\hline Hyperlipidemia & $65.4 \%$ \\
\hline Smoking & $48.0 \%$ \\
\hline Coronary artery disease & $30.8 \%$ \\
\hline Stroke & $34.6 \%$ \\
\hline Recent HbA1c & $6.6 \pm 0.7 \%$ \\
\hline Body mass index & $27 \pm 1.9$ \\
\hline mRS on admission & $1.6 \pm 0.6$ \\
\hline Symptomatic at presentation & $96.3 \%$ \\
\hline \multicolumn{2}{|l|}{ Clinical presentations } \\
\hline Ischemic stroke & $74.1 \%$ \\
\hline TIA & $22.2 \%$ \\
\hline Syncope & $3.7 \%$ \\
\hline \multicolumn{2}{|l|}{ Carotid lesion characteristic } \\
\hline \multicolumn{2}{|l|}{ Side undergoing stenting } \\
\hline Right & $63.0 \%$ \\
\hline Left & $37.0 \%$ \\
\hline Stenosis & $83.7 \pm 4.5 \%$ \\
\hline \multicolumn{2}{|l|}{ Location } \\
\hline Carotid bifurcation & $85.2 \%$ \\
\hline Distal to carotid bifurcation & $14.8 \%$ \\
\hline$>50 \%$ contralateral carotid stenosis & $20.8 \%$ \\
\hline Lesion length, mm & $12.9 \pm 1.7$ \\
\hline
\end{tabular}

\section{Outcomes}

The primary outcomes measured included the occurrence of transient ischemic attack (TIA), minor stroke, major stroke, myocardial infarction, and death. The definition of TIA includes transient focal neurological symptoms without acute infarction on neuroimaging or without permanent clinical deficits. The definition of minor stroke is a focal neurological symptom with acute infarction per neuroimaging or clinical findings, with changes in the National Institutes of Health Stroke Scale (NIHSS) score from 1 to 4 . Changes in the NIHSS score of more than 4 points were classified as evidence of major stroke. Myocardial infarction is defined as a chest pain event with positive cardiac enzymes and associated changes on electrocardiogram. Other postprocedural complications, including groin hematoma and hyperperfusion syndrome, were also recorded.

The secondary outcomes measured included technical success rate, stent placement success rate, and procedure success rate. Technical success is defined as the ability to successfully place the protection device, to establish flow reversal, and to retrieve the device. Stent placement success is defined as the ability to deploy the stent at the designated location. Procedure success is the ability to utilize the protection device without the occurrence of death or stroke. As previously mentioned, several factors were found to be associated with a higher percentage of adverse outcomes following carotid stenting with any protection device. The primary outcomes for each of these high-risk groups were recorded. The complication rate and procedural duration were compared between the long carotid lesion group and the control group (those who had shorter carotid lesions). Patients with contralateral carotid stenosis $>50 \%$, which predicts higher risk for a contralateral embolic event, were also evaluated. Statistical analysis was performed using the SPSS statistical data analysis software (version 22; IBM Corp., Armonk, N.Y., USA). The t test was used when appropriate. Statistical significance was set at $\mathrm{p}<0.05$. 
Table 2. Procedural data of the 27 patients and outcomes of CAS with a proximal protection device

Atchaneeyasakul et al.: Safety Outcomes Using a Proximal Protection Device in Carotid Stenting of Long Carotid Stenoses

\begin{tabular}{lc} 
Procedural data & \\
Procedure duration, min & $139.3 \pm 18.9$ \\
Stent size & $11.1 \%$ \\
$5 \mathrm{~mm}$ & $37.0 \%$ \\
$6 \mathrm{~mm}$ & $37.0 \%$ \\
$7 \mathrm{~mm}$ & $14.8 \%$ \\
$\quad$ Unknown & $117.1 \pm 15.4$ \\
Time to stent placement, min & \\
\hline Primary outcome & $0.0 \%$ \\
Death & $0.0 \%$ \\
Major stroke & $3.7 \%$ \\
Minor stroke & $0.0 \%$ \\
mRS worsening from admission & $0.0 \%$ \\
Cardiovascular event & $7.4 \%$ \\
Groin hematoma & $22.2 \%$ \\
Hyperperfusion syndrome & \\
\hline Secondary outcome & $100.0 \%$ \\
Technical success rate & $100.0 \%$ \\
Stent placement success rate & $96.3 \%$ \\
Procedure success rate & \\
\hline
\end{tabular}

\section{Results}

\section{Patient Information}

Retrospective data collection, which was performed from January 2011 to December 2014, identified 27 patients who had undergone CAS using a proximal protection device. All of those patients were included in the study. The patients' demographic data and medical histories are summarized in table 1 . Twenty-six patients $(96.3 \%)$ were symptomatic, as they presented with symptoms of acute stroke $(22 / 26 ; 84.6 \%)$ or TIA $(4 / 26 ; 15.4 \%)$. One patient $(3.7 \%)$ was asymptomatic. Patients previously diagnosed with hypertension, diabetes mellitus, and hyperlipidemia accounted for $92.3,34.6$, and $65.4 \%$, respectively. A history of smoking within the past 2 years was identified in $48 \%$ of our patients. A history of coronary artery disease and stroke was identified in 30.8 and $34.6 \%$, respectively. The average mRS score was $1.6 \pm 0.6$ at baseline.

\section{Carotid Lesion Characteristics}

The characteristics of the ICA lesions that underwent carotid stenting with a proximal embolic protection device are summarized in table 1 . The average percentage of carotid stenosis was $83.7 \pm 4.5 \%$. Near-occlusion ICA featuring the string sign was seen on angiography in 3 patients. The majority of stenoses were identified at the carotid bifurcation $(85.2 \%)$; this was in contrast to a minority of patients $(14.8 \%)$ who had a lesion distal to bifurcation. The average lesion length was $12.9 \pm 1.7 \mathrm{~mm}$. No ulcerated plaques were seen in any of the patients.

\section{Procedural Data and Outcomes}

Table 2 summarizes the procedure details and outcomes. The average procedure duration was $139.3 \pm 18.9 \mathrm{~min}$. There were no major coronary events, major stroke events, or deaths. One minor stroke event was recorded in a 73-year-old male who presented with an angiographic string sign of the carotid lesion. He developed dysarthria with worsening of 
his right-sided facial palsy, which was equivalent to a 3-point increase in the NIHSS score within $24 \mathrm{~h}$ after the procedure. The patient recovered completely from the stroke before being discharged. Postprocedural mRS scores were unchanged in the entire patient cohort. There was a technical success rate of $100 \%$ for carotid stent placement using the proximal protection device.

\section{High-Risk Lesion Subgroup Analysis}

High-risk carotid lesion length $>10 \mathrm{~mm}$ and/or angiographic string sign was noted in 21 out of 27 patients (77.8\%), averaging $14.4 \pm 1.6 \mathrm{~mm}$. The average carotid stenosis percentage in this group was $84.2 \pm 5.5 \%$. No patients in this high-risk group developed coronary syndrome or underwent a major ischemic stroke event. One in 21 patients (4.8\%) developed a minor stroke event. There was no significant difference in the complication rate or procedure duration between the long lesion group and the control group ( $p>0.05)$. Patients with significant stenosis of the contralateral carotid artery did not appear to have any periprocedural stroke events. The adverse event rates of other high-risk groups were $0 \%(0 / 7)$ in females and $14.3 \%(1 / 7)$ in those aged $>70$ years. No mortality was recorded in any of the high-risk groups.

\section{Discussion}

Embolic protection devices are widely utilized with carotid stenting due to a significant reduction in periprocedural strokes. Newly developed proximal embolic protection devices appear to be favorable when compared to distal embolic protection devices in several studies, as the former utilize internal artery flow reversal methods by occluding the ECA and CCA, thus preventing microemboli from dislodging distally [4]. Montorsi et al. [4] compared the rate of cerebral microembolization during CAS with a proximal cerebral protection device versus a distal cerebral protection device in patients with high-risk, lipid-rich plaques; the authors found a significantly lower microembolization rate in those patients undergoing CAS with a proximal protection device.

Our results showed a low percentage of adverse outcomes, as only 1 out of 27 patients (3.7\%) developed a minor stroke postprocedurally. Major stroke events were not seen in any of the patients. Our results are consistent with the findings of the ARMOUR trial, which evaluated the safety and effectiveness of proximal protection devices [12]. That study showed an adverse cardiovascular event rate of $2.7 \%$ and a major stroke rate of $0.9 \%$. Another objective of this study was to evaluate outcomes in a high-risk subgroup patient population that has not been very well studied. Mathur et al. [7] demonstrated that by using traditional stenting techniques, patients with long and/or multiple carotid lesions are at risk for minor and major stroke following the procedure, with an occurrence rate of up to $10 \%$ (1.1\% major stroke). Sayeed et al. [13] further showed that carotid lesion length is the strongest predictor for a stroke 30 days after carotid stenting with distal protection device usage. In our study, only $4.8 \%$ of the patients with a long carotid lesion (>10 mm) and/or angiographic string sign developed a minor stroke event. There was no report of acute coronary or major stroke events. The likely explanation for the higher stroke rate following distal embolic protection device usage as compared to the proximal protection device is the need to deploy the device distally to the lesion, which may be associated with a risk of dislodging atherosclerotic debris. This is highlighted by the fact that the filter traversed through the long lesion, which is not required with the proximal protection device. Complication rates and procedural duration were not different in the long carotid lesion group when compared to the control group. Furthermore, $100 \%$ technical and stent 
placement success rates were achieved. This illustrates how a proximal embolic protection device may represent a safe and suitable choice for use with carotid stenting in this group of patients.

It should be noted that there is another established high-risk characteristic in patients undergoing carotid stenting: with contralateral carotid stenosis $(>50 \%)$, proximal embolic protection device usage in our study also showed favorable outcomes with no coronary or stroke events in this group.

The role of angioplasty in patients undergoing stenting with proximal protection is no different from that with distal protection. Pre-angioplasty is performed so that the lesion can be traversed without disturbing the plaque and causing distal embolism. With complete arrest of integrated flow, pre-angioplasty in this case should be safer than with distal protection; the same applies to post-angioplasty. Based on our experience, the key is to not deflate the balloons immediately following angioplasty. Instead, one should aspirate blood through the central lumen until at least two consecutive aspirates are clear of debris. The ECA balloon should be deflated first, followed by the CCA balloon.

Several limitations are presented in this study, including the small sample size, the use of a single center, and the retrospective design. These elements should be further investigated in a multicenter, prospective study featuring a larger patient population.

\section{Conclusion}

Proximal embolic protection devices are safe for patients with carotid stenosis. They are also safe for those patients with a carotid lesion length $>10 \mathrm{~mm}$ and/or angiographic string sign, as observed in our patient cohort.

\section{Disclosure Statement}

None of the authors reports any financial disclosure relevant to this paper.

\section{References}

1 Go AS, Mozaffarian D, Roger VL, Benjamin EJ, Berry JD, Blaha MJ, Dai S, Ford ES, Fox CS, Franco S, Fullerton HJ, Gillespie C, Hailpern SM, Heit JA, Howard VJ, Huffman MD, Judd SE, Kissela BM, Kittner SJ, Lackland DT, Lichtman JH, Lisabeth LD, Mackey RH, Magid DJ, Marcus GM, Marelli A, Matchar DB, McGuire DK, Mohler ER 3rd, Moy CS, Mussolino ME, Neumar RW, Nichol G, Pandey DK, Paynter NP, Reeves MJ, Sorlie PD, Stein J, Towfighi A, Turan TN, ViraniSS, Wong ND, Woo D, Turner MB; American Heart Association Statistics Committee and Stroke Statistics Subcommittee: Heart disease and stroke statistics - 2014 update: a report from the American Heart Association. Circulation 2014;129:e28-e292.

2 Perkins WJ, Lanzino G, Brott TG: Carotid stenting vs endarterectomy: new results in perspective. Mayo Clin Proc 2010;85:1101-1108.

3 SPACE Collaborative Group, Ringleb PA, Allenberg J, Brückmann H, Eckstein HH, Fraedrich G, Hartmann M, Hennerici M, Jansen O, Klein G, Kunze A, Marx P, Niederkorn K, Schmiedt W, Solymosi L, Stingele R, Zeumer H, Hacke W: 30 day results from the SPACE trial of stent-protected angioplasty versus carotid endarterectomy in symptomatic patients: a randomised non-inferiority trial. Lancet 2006;368:1239-1247.

4 Montorsi P, Caputi L, Galli S, Ciceri E, Ballerini G, Agrifoglio M, Ravagnani P, Trabattoni D, Pontone G, Fabbiocchi F, Loaldi A, Parati E, Andreini D, Veglia F, Bartorelli AL: Microembolization during carotid artery stenting in patients with high-risk, lipid-rich plaque. A randomized trial of proximal versus distal cerebral protection. J Am Coll Cardiol 2011;58:1656-1663.

5 Schmidt A, Diederich KW, Scheinert S, Bräunlich S, Olenburger T, Biamino G, Schuler G, Scheinert D: Effect of two different neuroprotection systems on microembolization during carotid artery stenting. J Am Coll Cardiol 2004;44:1966-1969. 
6 Bijuklic K, Wandler A, Hazizi F, Schofer J: The PROFI study (Prevention of Cerebral Embolization by Proximal Balloon Occlusion Compared to Filter Protection During Carotid Artery Stenting): a prospective randomized trial. J Am Coll Cardiol 2012;59:1383-1389.

7 Mathur A, Roubin GS, Iyer SS, Piamsonboon C, Liu MW, Gomez CR, Yadav JS, Chastain HD, Fox LM, Dean LS, Vitek JJ: Predictors of stroke complicating carotid artery stenting. Circulation 1998;97:1239-1245.

8 Eckstein HH, Ringleb P, Allenberg JR, Berger J, Fraedrich G, Hacke W, Hennerici M, Stingele R, Fiehler J, Zeumer $\mathrm{H}$, Jansen O: Results of the Stent-Protected Angioplasty versus Carotid Endarterectomy (SPACE) study to treat symptomatic stenoses at 2 years: a multinational, prospective, randomised trial. Lancet Neurol 2008;7:893902.

9 Brott TG, Hobson RW 2nd, Howard G, Roubin GS, Clark WM, Brooks W, Mackey A, Hill MD, Leimgruber PP, Sheffet AJ, Howard VJ, Moore WS, Voeks JH, Hopkins LN, Cutlip DE, Cohen DJ, Popma JJ, Ferguson RD, Cohen SN, Blackshear JL, Silver FL, Mohr JP, Lal BK, Meschia JF; CREST Investigators: Stenting versus endarterectomy for treatment of carotid-artery stenosis. N Engl J Med 2010;363:11-23.

10 Fox AJ: How to measure carotid stenosis. Radiology 1993;186:316-318.

11 Barnett HJ, Taylor DW, Eliasziw M, Fox AJ, Ferguson GG, Haynes RB, Rankin RN, Clagett GP, Hachinski VC, Sackett DL, Thorpe KE, Meldrum HE, Spence JD: Benefit of carotid endarterectomy in patients with symptomatic moderate or severe stenosis. North American Symptomatic Carotid Endarterectomy Trial Collaborators. N Engl J Med 1998;339:1415-1425.

12 Ansel GM, Hopkins LN, Jaff MR, Rubino P, Bacharach JM, Scheinert D, Myla S, Das T, Cremonesi A; Investigators for the ARMOUR Pivotal Trial: Safety and effectiveness of the INVATEC MO.MA proximal cerebral protection device during carotid artery stenting: results from the ARMOUR pivotal trial. Catheter Cardiovasc Interv 2010; 76:1-8.

13 Sayeed S, Stanziale SF, Wholey MH, Makaroun MS: Angiographic lesion characteristics can predict adverse outcomes after carotid artery stenting. J Vasc Surg 2008;47:81-87. 